\title{
Correlation of ulcerative colitis and colorectal cancer: a systematic review and meta-analysis
}

\author{
Yansong Wang, Pu Wang, Lixiang Shao \\ Department of Oncology, The General Hospital of Western Theater Command, Chengdu, China \\ Contributions: (I) Conception and design: Y Wang, P Wang; (II) Administrative support: L Shao; (III) Provision of study materials or patients: Y \\ Wang, P Wang; (IV) Collection and assembly of data: All authors; (V) Data analysis and interpretation: All authors; (VI) Manuscript writing: All \\ authors; (VII) Final approval of manuscript: All authors. \\ Correspondence to: Yansong Wang. Department of Oncology, The General Hospital of Western Theater Command, 270 Tianlu Road, Rongdu Dadao, \\ Chengdu, China. Email: wangyansong_xz@163.com.
}

\begin{abstract}
Background: A large number of studies have shown that ulcerative colitis (UC) can increase the risk of colorectal cancer (CRC). The purpose of the present study was to explore the specific mechanism of UC influence on CRC.

Methods: We searched PubMed for articles related to CRC and colitis since the establishment of the database until April 2021. Keywords, such as ulcerative colitis, colorectal cancer, and relevance, were used for the article search. Two investigators read through the full text according to the inclusion and exclusion criteria to screen the articles. Cochrane system review manual (version 5.3) was adopted to evaluate the quality of the selected articles. Then, data was extracted, and the overall risk of UC patients into CRC, the course of the disease, and the region were systematically analyzed.

Results: A total of 11 studies involving 26,765 patients with UC were included. The results showed that $\mathrm{UC}$ is one of the risk factors for CRC. we also found that geographical location also had an impact on the transition from UC to CRC, but the impact was not significant. Patients with colitis had a significantly higher rate of conversion to CRC after 10 to 20 years of disease.

Discussion: A total of 11 articles were included to analyze the association between UC and CRC. The studies found that the location, duration, and geographical location of patients with UC directly affected the occurrence of CRC and are independent risk factors for the transformation of UC into CRC.
\end{abstract}

Keywords: Ulcerative colitis (UC); colorectal cancer (CRC); independent risk factors; meta-analysis

Submitted Sep 09, 2021. Accepted for publication Oct 29, 2021.

doi: 10.21037/jgo-21-624

View this article at: https://dx.doi.org/10.21037/jgo-21-624

\section{Introduction}

Ulcerative colitis (UC) is an inflammatory disease of the rectum. To date, its etiology is still unknown. The lesion site is mostly concentrated in the colonic mucosa and often involves the distal colon, further developing to the proximal end. Its main clinical symptoms are abdominal pain, diarrhea, mucus pus, and bloody stools. UC shows no significant course change and often alternates between aggravation and remission (1). This has a significant impact on the diagnosis and treatment of the disease. The incidence of colorectal cancer (CRC) in western countries is about $1 \%$. In recent years, the incidence of UC in China has been rising annually because of the increase in Western diets and living habits (2). CRC is a common tumor of the digestive tract. In recent years, its status on the global cancer spectrum has been increasing, ranking third among new malignant tumors and cancer deaths in the USA in 2007. In China, CRC has been one of the fastest rising malignancies in the past two decades, with 146,000 new cases and 78,700 deaths each year. It is the fifth leading cause of death in China and the fourth leading cause of cancer-related death (3). A large number of 
clinical data show that patients with $\mathrm{UC}$ are more likely to develop CRC. Current studies have identified UC as one of the major independent risk factors for CRC (4). Other studies have shown that as patients with $\mathrm{UC}$ age, their risk of developing CRC increases significantly. These findings indicate that there is a correlation between $\mathrm{UC}$ and the occurrence of CRC (5); therefore, CRC can be considered a serious complication of UC. Clinical data show that UCrelated CRC accounts for $5 \%$ of the total number of CRC cases. Generally speaking, the occurrence and development of colonic cancer are the following classic pattern of "mutation accumulation-adenoma-canceration".

The adenomatous polyposis coli $(A P C)$ gene, a tumorsuppressor gene, was first discovered on the chromosome of a patient with rectal tumor and mental disability. The classical theory of CRC suggests that APC occurs in the early stage of cancer. However, the pathogenesis of UC-associated CRC is different from that of classic CRC (6). For example, it has been reported that $A P C$ mutations occur late, while p 53 mutations are early events in the carcinogenesis process. Therefore, research on UC-related CRC has become a hot spot in the field of intestinal diseases. However, due to the lack of knowledge of the etiology of UC-related CRC, it is unclear how UC specifically affects the occurrence and development of CRC (7).

The first retrospective study of the occurrence and progression of CRC was published in 1988. In that study, the incidence of CRC in UC was reported to be $4.25 \%$ (8). In recent years, data from relevant worldwide studies have shown that the standardized incidence ratio (SIR) of CRC associated with UC may be related to the course of the patient's disease and regional differences. For example, the cumulative rate of CRC diagnosis 10 years after UC diagnosis was reported to be $4.9 \%$ (9). The risk of CRC was not found to be higher among US patients compared with age- and sex-matched counterparts in the general population. However, the study did not take into account risk factors, such as disease scope, duration, and location. The relationship between UC and CRC has not been thoroughly explained (10). At present, there are many meta-analyses on the correlation between UC and CRC, but most of them only consider one influencing factor. There is no study of penalties from multiple perspectives. Based on previous related researches, this study conducted a meta-analysis of the correlation between UC and CRC from the perspectives of disease course, region, and overall conversion rate, to further promote the research in the frontier field.

We present the following article in accordance with the
PRISMA reporting checklist (available at https://dx.doi. org/10.21037/jgo-21-624).

\section{Methods}

\section{Literature search}

A comprehensive and systematic literature study was conducted based on the Cochrane Handbook for Systematic Reviews of Interventions, while following the PRISMA and MOOSE guidelines for meta-analyses of reports. We searched PubMed and scientific conferences, and identified articles on the correlation of UC and CRC. All of the studies included patients with CRC associated with UC. Articles were searched using keywords, such as UC, inflammatory bowel disease, and CRC. Studies of UC had to be associated with CRC. Incidence was also included in the studies as an indirect method to adjust for disease scope, disease course, and region.

\section{Inclusion and exclusion criteria}

Articles were included in the meta-analysis if they met the following inclusion criteria: (I) experimental data contained the required basic information; (II) CRC associated with UC; (III) the number of cases was sufficient and the sample size was large enough; (IV) clinical trials; and (V) published articles.

Articles were excluded from the meta-analysis if they met any of the following exclusion criteria: (I) CRC not associated with UC; (II) meta-analyses or reviews; (III) literature with incomplete data and unable to obtain further information; (IV) results were not CRC; (V) the disease types studied in the literature were Crohn's disease, community acquired pneumonia (CAP), family history of CRC, or UC colectomy; and (VI) the literature was duplicated and republished.

\section{Literature screening}

Two researchers first screened the titles and abstracts independently according to the qualified criteria, and then extracted the data and evaluated the quality. If the evaluation results were inconsistent, other researchers were consulted to resolve the discrepancy. If the title and abstract of the article met the literature requirements, the full text was retrieved for data extraction. NoteExpress 2.0 (Beijing Love Sea Software Company) was used for 
literature management; duplicate literature was deleted. The inclusion of literature was checked according to the criteria mentioned above.

\section{Data extraction}

The 2 researchers independently extracted relevant information from all eligible studies using a predefined data extraction table as follows: author, year of publication, sample size, age, country, sex, degree of disease, and course of disease. For missing data, the researchers contacted the original authors of the literature via email.

\section{Quality assessment}

To improve the quality of the reviewed literature, the quality was assessed in accordance with the risk of bias assessment recommended in version 5.3 of the Cochrane Handbook for Systematic Reviews of Interventions. The evaluation included the following: (I) which random method to use; (II) whether the grouping condition is hidden; (III) the implementation of the blind method between patients and researchers; (IV) evaluate the effect of the blind method. Is there a result the integrity of the credibility of 7 other 6 survey bias about the item 7 of the randomized controlled trials, "meet" indicates bias is small, "not satisfied" refers to height bias, study is not fully detailed report, if no mention, danger is unknown evaluation including random sequence generation, allocation concealment, blinded.

A score of $1-3$ in the 4 dimensions of tracking/exit is considered to be low quality, and a score of $4-7$ is considered to be high quality.

\section{Data extraction}

The forest map also makes clear the results of individual studies, combining those studies with corresponding confidence intervals (CIs). If there is no overlap between the CIs of the individual studies, this indicates statistical inhomogeneity between the studies. Further subgroup analysis is required to combine stochastic and fixed models with acceptable inhomogeneity. There is heterogeneity/ inhomogeneity in the research, and the heterogeneity/ inhomogeneity can be reduced by setting the subgroup analysis, and the combined effect value can be calculated. Sensitivity analysis: after adjusting the individual studies, whether it can affect the overall results is explored. If the overall results are affected, it indicates that there is sensitivity. Generally speaking, this study believes that it will have an impact on the comprehensive study under the following two circumstances. If the documents that do not meet the requirements are deleted, the results yield significantly different results. If a study affects the overall results with little difference, this indicates the sensitivity of the combined results and the results obtained are not stable. On the contrary, the results show that the sensitivity is stable and the meta results obtained are trustworthy.

\section{Statistical analysis}

A randomized effects meta-analysis was used to evaluate the incidence of CRC in patients with UC. To calculate the combined SIR for CRC, we combined the extracted study specificity estimates with $95 \%$ CIs using the DerSimonianLaird random-effects model. Publication bias (small study effect) was detected by symmetry of the funnel plot of visual assessment; asymmetry was assessed by BeggMazumdar's rank test. Forest maps were developed for the prevalence of the study results in the population and within the group. $\mathrm{R}$ software (version 3.4.4) was used for data processing and statistical analysis. The calculation method used odds ratio (OR) as the effect size, and 95\% CI was used to express the result. The included studies were tested for heterogeneity first, with $\alpha=0.1$ as the test level. If there was no heterogeneity between the studies $(\mathrm{P}>0.1$, $\left.\mathrm{I}^{2}<50 \%\right)$, the fixed effects model was selected for metaanalysis. Otherwise, subgroup analysis was performed on the included data. $\mathrm{P}<0.05$ indicated that the difference was statistically significant.

\section{Results}

\section{Literature search results}

A total of 4,778 related articles were retrieved in this study, including 1,086 from PubMed, 1,177 from Embase, 991 from Ovid MEDLINE and 1,524 from registers. After reading the title and abstract, 3,491 studies that did not meet the inclusion criteria were excluded. After reading the full text, 1,267 studies were excluded and 11 studies that met the inclusion criteria were included (11-21). The literature screening process is detailed in Figure 1 and Table 1.

\section{Risk of bias assessment of included articles}

The Cochrane Handbook for Systematic Reviews of 


\section{Identification of studies via databases and registers}

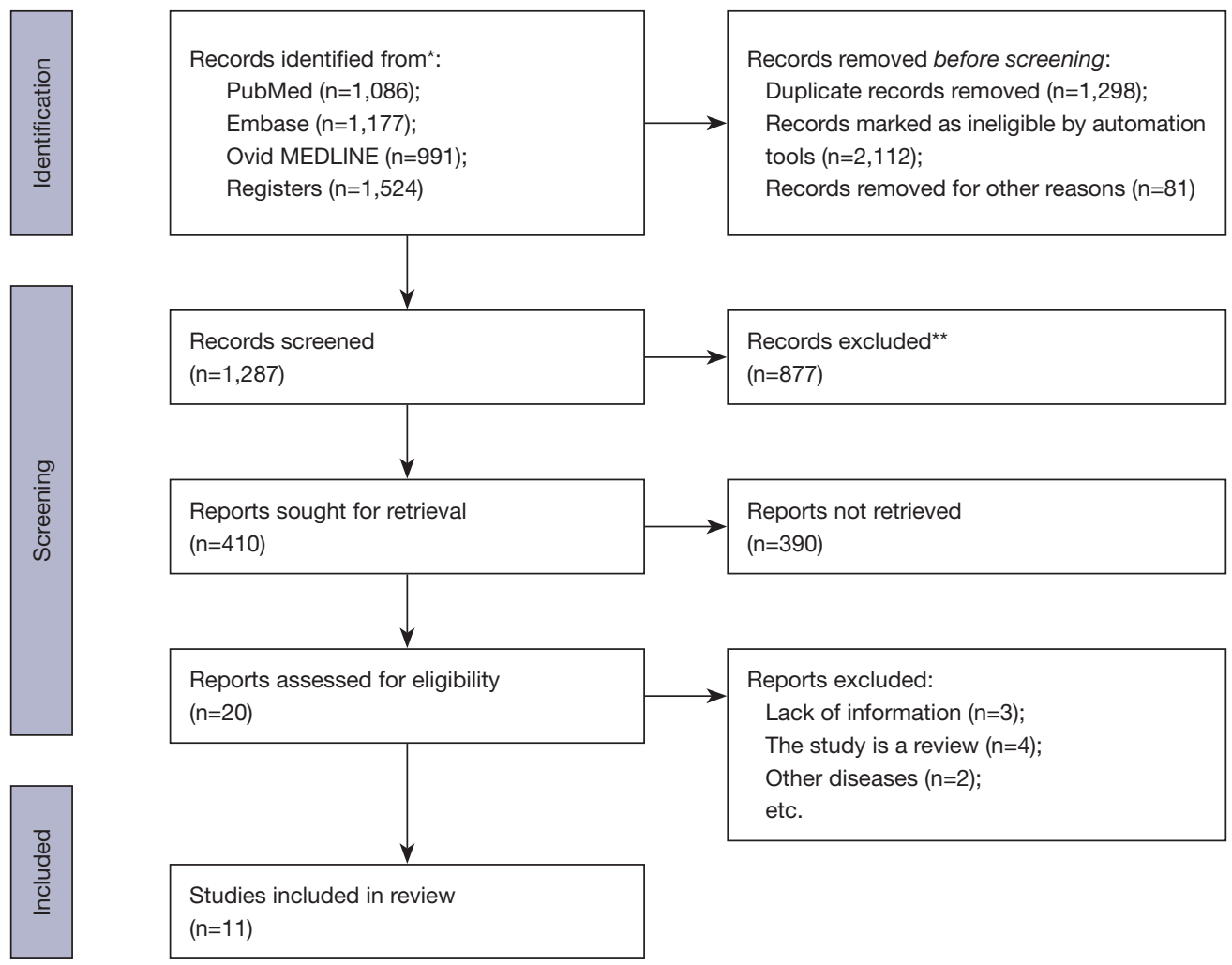

Figure 1 Literature retrieval process. *, consider, if feasible to do so, reporting the number of records identified from each database or register searched (rather than the total number across all databases/registers); ${ }^{* *}$, if automation tools were used, indicate how many records were excluded by a human and how many were excluded by automation tools.

Table 1 Basic characteristics of the included articles

\begin{tabular}{lccccc}
\hline First author & Year & Country & Ulcerative colitis (case) & Colorectal cancer (case) & Incidence of colorectal cancer (\%) \\
\hline Desai (11) & 2015 & India & 430 & 12 & 2.80 \\
Shivakumar (12) & 2013 & India & 29 & 5 & 3.40 \\
Venkataraman (13) & 2005 & India & 532 & 26 & 0.94 \\
Kim (14) & 2009 & South Korea & 7,061 & 0 & 0.37 \\
Hilmi (15) & 2009 & Malaysia & 118 & 0 & 0.00 \\
Al-Shamali (16) & 2003 & Kuwait & 346 & 3 & 0.00 \\
Kekilli (17) & 2010 & Turkey & 275 & 25 & 1.10 \\
Kamiya (18) & 2015 & Japan & 1,583 & 4 & 0.64 \\
Zhang (19) & 2015 & China & 624 & 26 & 2.51 \\
Gilat (20) & 1988 & Israel & 1,035 & 2 & 0.50 \\
Senanayake (21) & 2013 & Sri Lanka & 3,428 & & 0 \\
\hline
\end{tabular}




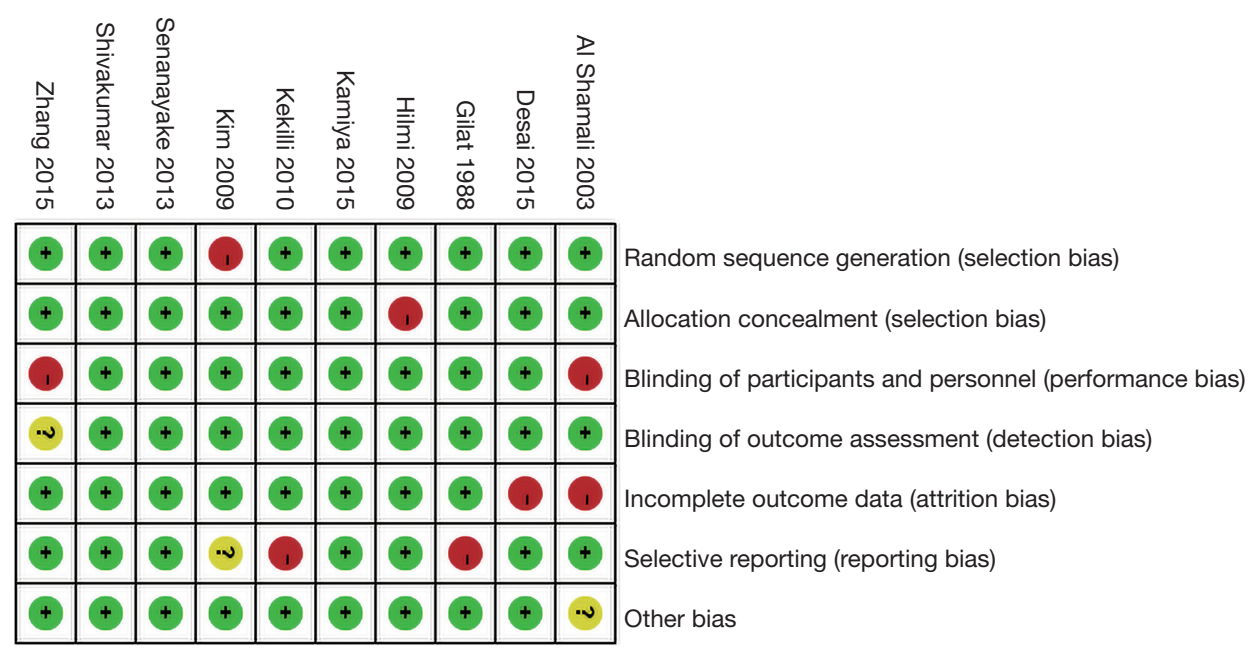

Figure 2 Risk of bias assessment of the included articles.

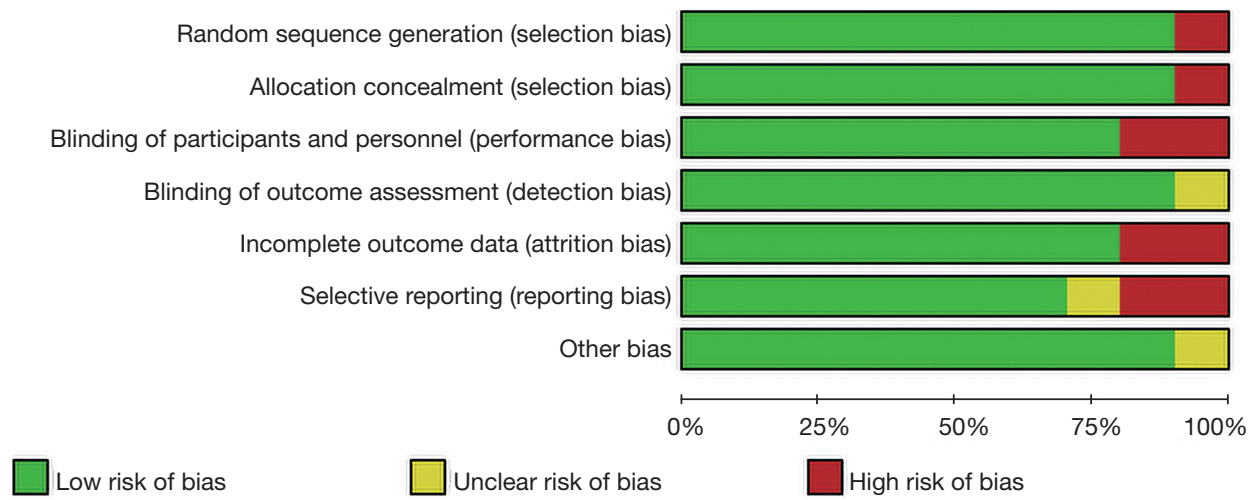

Figure 3 Bias evaluation of the included articles.

Interventions (version 5.0.2) was used to evaluate the risk of bias in the 8 articles included in this study. Review Manager 5.3 was employed to create the risk of bias chart (Figures 2,3).

\section{Overall risk of CRC in patients with $U C$}

A total of 11 articles met the inclusion criteria. The overall risk of CRC in patients with UC was analyzed. The results showed that $\mathrm{I}^{2}=89 \%, \mathrm{P}<0.00001$. According to this, $\mathrm{I}^{2}=89 \%$ is greater than $50 \%$, so the random-effects model was selected. There was heterogeneity RR (95\% CI): 0.01 (0.01 to 0.01$), Z=47.86, P<0.00001$, and the analysis showed a difference in the number of patients with $\mathrm{UC}$ alone and those with UC + inflammatory CRC (Figure 4). This suggests that $\mathrm{UC}$ is a risk factors for CRC. The funnel plot is symmetric, and most of the data are on both sides of the central axis (Figure 5). This suggests that publication bias was effective. It can be concluded that UC is a major independent risk factor for CRC.

\section{Meta-analysis of course of progression from UC to CRC}

The course analysis of patients with UC converting to CRC is shown in Table 2. Table 1 shows that after 10 to 20 years of ulcerative colon cancer, the probability of transforming to colon cancer increases significantly (Table 2).

\section{Meta-analysis of regional differences in patients with $U C$ transforming to CRC in Asia}

The results of regional differences in the conversion of patients with UC to CRC in Asia are shown in Table 3. 


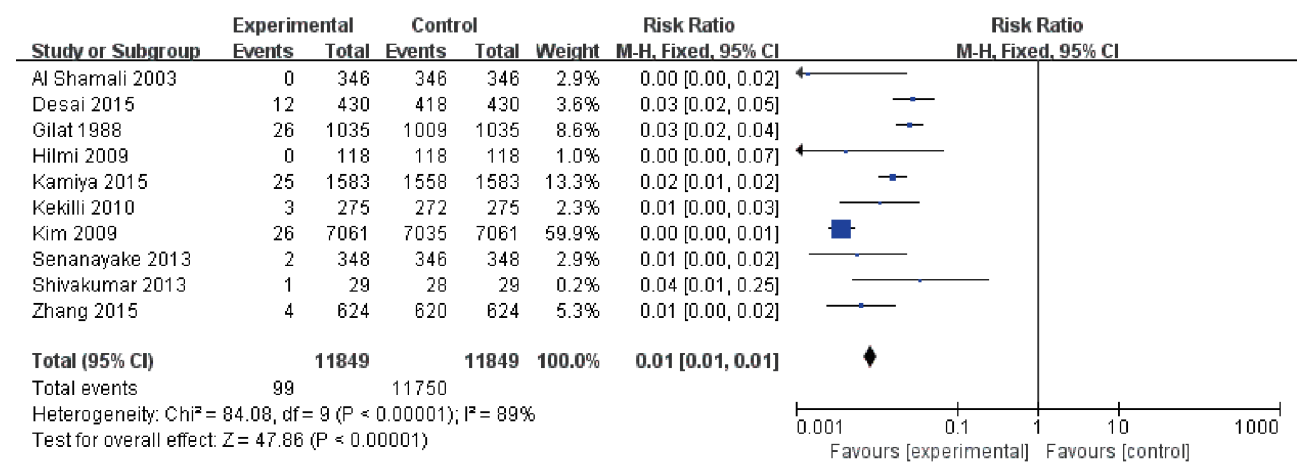

Figure 4 Forest plot for the number of patients with ulcerative colitis which progressed to colorectal cancer. CI, confidence interval; M-H, Mantel-Haenszel.

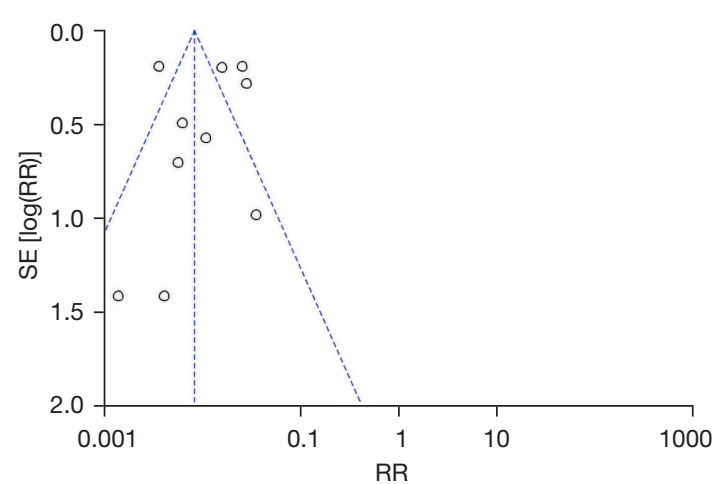

Figure 5 Funnel plot the number of patients with ulcerative colitis which transformed to colorectal cancer. RR, relative risk; SE, standard error.

According to Table 3, there were differences in the CRC conversion rate among patients with UC in different regions in Asia, but the difference was not statistically significant. UC patients in South Asia had the highest CRC conversion rate (Table 3).

\section{Discussion}

CRC is a major long-term serious complication in patients with UC (22-24). Previous studies have shown that the main area of colon cancer incidence $(25,26)$. Although recent studies have suggested that patients with UC do not have a particularly high rate of CRC conversion, the prevalence is still very high compared with the general population $(27,28)$. Numerous studies have suggested ethnic differences in the incidence of UC, with most previous statistical studies focusing on European regions (29). Further studies on the transition from UC to CRC in Asia, it is of great significance to understand the correlation between UC and colon cancer.

In the present study, we analyzed the transformation of UC to CRC in Asian patients, considering disease range, course, and regional differences. The findings are consistent with reports from European countries that UC is strongly associated with CRC (30). The overall risk analysis of UC patients in Asia was 95\% CI: 0.01-0.01 $(\mathrm{Z}=47.86, \mathrm{P}<0.00001)$. This suggests that $\mathrm{UC}$ is an important independent risk factor for CRC in Asia. In the study of the effect of the course of colitis on the incidence of colon cancer, we found that the course of the disease has a greater impact on the incidence of colon cancer in patients with ulcerative colon inflammation in Asian countries. The incidence of CRC was significantly increased in patients with UC for more than 20 years of illness. Analysis of regional differences in the incidence of CRC in patients with UC in Asian countries showed that there were slight differences in the incidence of CRC in patients with UC in different regions of Asia, but the differences were not particularly significant. South Asia had the highest incidence, but the differences were not significant. Overall, the results of the present study indicate that UC is an important independent risk factor for CRC in Asia. However, the duration of the disease in patients with $\mathrm{UC}$ is a major factor affecting the transition to CRC. The impact of regional differences on the transition to CRC in Asian patients with UC was not significant.

All of the articles included in this study were about the number of patients with UC, the number of patients with CRC, the region of patients, and the course of the disease. There were some differences in the grouping. Two articles 
Table 2 Results of course analysis of patients with ulcerative colitis converting to colorectal cancer

\begin{tabular}{lcccc}
\hline Years of disease (years) & Article $(\mathrm{n})$ & Conversion rate $(\%)$ & $95 \%$ confidence interval & Proportion (\%) \\
\hline $1-9$ & 1 & 0.069 & $0.004-0.008$ & 23.8 \\
$10-20$ & 6 & 0.012 & $0.01-0.015$ & 36.5 \\
$21-30$ & 2 & 0.019 & $0.013-0.025$ & 15.0 \\
$\geq 30$ & 2 & 0.016 & $0.012-0.021$ & 25.3 \\
\hline
\end{tabular}

Table 3 Basic characteristics of the included literature

\begin{tabular}{lccc}
\hline Region & Articles $(\mathrm{n})$ & Conversion rate $(\%)$ & $95 \%$ confidence interval \\
\hline Asia & 11 & 0.78 & $0.29-1.21$ \\
East Asia & 3 & 0.69 & $0.49-0.90$ \\
South Asia & 2 & 1.28 & $0.83-1.81$ \\
Middle East & 3 & 0.84 & $0.29-1.38$ \\
West Asia & 3 & 0.65 & $0.48-0.81$ \\
\hline
\end{tabular}

were grouped according to the region of the patients, 6 articles were grouped according to different disease types, and 3 articles were grouped according to different disease course. All of the articles included in this study were on the transformation of UC to CRC in Asia, and data from other continents such as Europe and North America are not included. Therefore, it may have a certain impact on the results of this article.

\section{Conclusions}

In the present study, we analyzed the transformation of UC to CRC from the perspectives of scope, course, and regional differences. The findings indicated that $\mathrm{UC}$ is an independent risk factor for CRC in Asia, and the findings were consistent with those of European studies. The course of disease in patients with UC has a significant effect on its transformation to CRC. The influence of regional difference on the transformation of UC to CRC was not obvious.

\section{Acknowledgments}

Funding: None.

\section{Footnote}

Reporting Checklist: The authors have completed the
PRISMA reporting checklist. Available at https://dx.doi. org/10.21037/jgo-21-624

Conflicts of Interest: All authors have completed the ICMJE uniform disclosure form (available at https://dx.doi. org/10.21037/jgo-21-624). The authors have no conflicts of interest to declare.

Ethical Statement: The authors are accountable for all aspects of the work in ensuring that questions related to the accuracy or integrity of any part of the work are appropriately investigated and resolved.

Open Access Statement: This is an Open Access article distributed in accordance with the Creative Commons Attribution-NonCommercial-NoDerivs 4.0 International License (CC BY-NC-ND 4.0), which permits the noncommercial replication and distribution of the article with the strict proviso that no changes or edits are made and the original work is properly cited (including links to both the formal publication through the relevant DOI and the license). See: https://creativecommons.org/licenses/by-nc-nd/4.0/.

\section{References}

1. Kucharzik T, Koletzko S, Kannengiesser K, et al. Ulcerative Colitis-Diagnostic and Therapeutic Algorithms. Dtsch Arztebl Int 2020;117:564-74. 
2. Keshteli AH, Madsen KL, Dieleman LA. Diet in the Pathogenesis and Management of Ulcerative Colitis; A Review of Randomized Controlled Dietary Interventions. Nutrients 2019;11:1498.

3. Wang R, Han L, Dai W, et al. Cause of death for elders with colorectal cancer: a real-world data analysis. J Gastrointest Oncol 2020;11:269-76.

4. Blanchaert C, Strubbe B, Peeters H. Fecal microbiota transplantation in ulcerative colitis. Acta Gastroenterol Belg 2019;82:519-28.

5. Nanki K, Fujii M, Shimokawa M, et al. Somatic inflammatory gene mutations in human ulcerative colitis epithelium. Nature 2020;577:254-9.

6. Yamamoto-Furusho JK, Gutiérrez-Grobe Y, LópezGómez JG, et al. The Mexican consensus on the diagnosis and treatment of ulcerative colitis. Rev Gastroenterol Mex (Engl Ed) 2018;83:144-67.

7. Gallo G, Kotze PG, Spinelli A. Surgery in ulcerative colitis: When? How? Best Pract Res Clin Gastroenterol 2018;32-33:71-8.

8. Lopez A, Pouillon L, Beaugerie L, et al. Colorectal cancer prevention in patients with ulcerative colitis. Best Pract Res Clin Gastroenterol 2018;32-33:103-9.

9. Clarke WT, Feuerstein JD. Colorectal cancer surveillance in inflammatory bowel disease: Practice guidelines and recent developments. World J Gastroenterol 2019;25:4148-57.

10. Keller DS, Windsor A, Cohen R, et al. Colorectal cancer in inflammatory bowel disease: review of the evidence. Tech Coloproctol 2019;23:3-13.

11. Desai D, Shah S, Deshmukh A, et al. Colorectal cancers in ulcerative colitis from a low-prevalence area for colon cancer. World J Gastroenterol 2015;21:3644-9.

12. Shivakumar BM, Lakshmankumar B, Rao L, et al. Colorectal neoplasia in long-standing ulcerative colitis - a prospective study from a low-prevalence area. Colorectal Dis 2013;15:e462-8.

13. Venkataraman S, Mohan V, Ramakrishna BS, et al. Risk of colorectal cancer in ulcerative colitis in India. J Gastroenterol Hepatol 2005;20:705-9.

14. Kim BJ, Yang SK, Kim JS, et al. Trends of ulcerative colitis-associated colorectal cancer in Korea: A KASID study. J Gastroenterol Hepatol 2009;24:667-71.

15. Hilmi I, Singh R, Ganesananthan S, et al. Demography and clinical course of ulcerative colitis in a multiracial Asian population: a nationwide study from Malaysia. J Dig Dis 2009;10:15-20.

16. Al-Shamali MA, Kalaoui M, Patty I, et al. Ulcerative colitis in Kuwait: a review of 90 cases. Digestion 2003;67:218-24.

17. Kekilli M, Dagli U, Kalkan IH, et al. Low incidence of colorectal dysplasia and cancer among patients with ulcerative colitis: a Turkish referral centre study. Scand J Gastroenterol 2010;45:434-9.

18. Kamiya T, Ando T, Watanabe O, et al. Suitability of surveillance colonoscopy for patients with ulcerative colitis to detect colorectal cancer: current guidelines miss some early-stage cases. Nagoya J Med Sci 2015;77:237-44.

19. Zhang Q, Sha S, Xu B, et al. Prevalence of colorectal cancer in patients with ulcerative colitis: A retrospective, monocenter study in China. J Cancer Res Ther 2015;11:899-903.

20. Gilat T, Fireman Z, Grossman A, et al. Colorectal cancer in patients with ulcerative colitis. A population study in central Israel. Gastroenterology 1988;94:870-7.

21. Senanayake SM, Fernandopulle AN, Niriella MA, et al. The long-term outcomes of a cohort of Sri Lankan patients with ulcerative colitis: a retrospective study at two national referral centers and review of literature. Clin Exp Gastroenterol 2013;6:195-200.

22. Gajendran M, Loganathan P, Jimenez G, et al. A comprehensive review and update on ulcerative colitis. Dis Mon 2019;65:100851.

23. Olén O, Erichsen R, Sachs MC, et al. Colorectal cancer in ulcerative colitis: a Scandinavian population-based cohort study. Lancet 2020;395:123-31.

24. Yan P, Wang Y, Meng X, et al. Whole Exome Sequencing of Ulcerative Colitis-associated Colorectal Cancer Based on Novel Somatic Mutations Identified in Chinese Patients. Inflamm Bowel Dis 2019;25:1293-301.

25. Farrukh A, Mayberry JF. Surveillance for colorectal cancer and chemoprevention in ulcerative and Crohn's colitis: The need for clinical strategies to increase effectiveness. JGH Open 2019;3:370-3.

26. Yao D, Dong M, Dai C, et al. Inflammation and Inflammatory Cytokine Contribute to the Initiation and Development of Ulcerative Colitis and Its Associated Cancer. Inflamm Bowel Dis 2019;25:1595-602.

27. de Campos Silva EF, Baima JP, de Barros JR, et al. Risk factors for ulcerative colitis-associated colorectal cancer: A retrospective cohort study. Medicine (Baltimore) 2020;99:e21686.

28. Kvorjak M, Ahmed Y, Miller ML, et al. Cross-talk between Colon Cells and Macrophages Increases ST6GALNAC1 and MUC1-sTn Expression in Ulcerative Colitis and Colitis-Associated Colon Cancer. Cancer Immunol Res 2020;8:167-78. 
29. Tian Y, Xu Q, Sun L, et al. Short-chain fatty acids administration is protective in colitis-associated colorectal cancer development. J Nutr Biochem 2018;57:103-9.

30. Baker AM, Cross W, Curtius K, et al. Evolutionary

Cite this article as: Wang Y, Wang P, Shao L. Correlation of ulcerative colitis and colorectal cancer: a systematic review and meta-analysis. J Gastrointest Oncol 2021;12(6):2814-2822. doi: 10.21037/jgo-21-624 history of human colitis-associated colorectal cancer. Gut 2019;68:985-95.

(English Language Editor: R. Scott) 